\title{
Assessing the effects of underground mining activities on high-voltage overhead power lines
}

\author{
Vladimir Gusev ${ }^{1}$, Alexei Zhuravlyov ${ }^{2}$,Elena Maliukhina ${ }^{*}$ \\ ${ }^{1}$ Department of Mine Surveying, Saint-Petersburg Mining University, 2, 21st Line, St Petersburg \\ 199106, Russia \\ ${ }^{2}$ Jet Technology \& Construction Company, Dnepropetrovskaya st.,14 ,St. Petersburg 192007, Russia
}

\begin{abstract}
This paper introduces a technique for predictive assessment of changes in the position of power transmission towers and condition of overhead power lines, located in the zone of influence of displacements and deformations of the Earth's surface caused by mining activities. A special approach for monitoring the technical condition of towers and cables is proposed. It is intended to address the issue of controlling the condition of transmission lines that are under the influence of underground mining activities and to checkmate such impact.
\end{abstract}

\section{Introduction}

The issue of the effect of underground mining activities on overhead power transmission lines in the major coal basins in Russia is addressed by determining a safe depth for transmission towers $[1,2]$ situated in the zone of influence of the mining activities. The safe depth is calculated using the formula:

$$
H_{s}=K_{\sigma} \cdot m,
$$

where $m$ is the mineable seam thickness, $K_{s}$ is the safety factor, which depends on the type of tower (anchor-angle, intermediate angle and straight, dead-end towers) and the voltage of the power transmitted $\left(\mathrm{K}_{\mathrm{s}}=60 \div 100\right)$. Layers under transmission towers can be excavated at a depth no less than a safe depth calculated by formula (1). The conditions for such formations are given in [1]. It is necessary to take protective measures when conducting mining activities whose depth goes beyond the safe depth. For transmission towers situated in the zone of influence of subway tunnel construction, the mineable seam thickness in formula (1) can be replaced by the sum of displacements of the tunnel arch crown before tunnel fixing and displacements of the hinge part of the crown after tunnel fixing. We now proceed further as in $[1,2]$.

\section{Research techniques}

Since transmission towers situated within the boundaries of the zone of influence of underground mining or construction of underground subway facilities will be affected by displacements and deformations (Fig. 1), there is need to level individual poles and re-stretch

\footnotetext{
*Corresponding author: elenamaliukhina@gmail.com
} 
the cables and wires to normal state. This is regardless of whether the mining or subway tunnel construction will be carried out above or below the safe depth horizon.

$S_{1}$

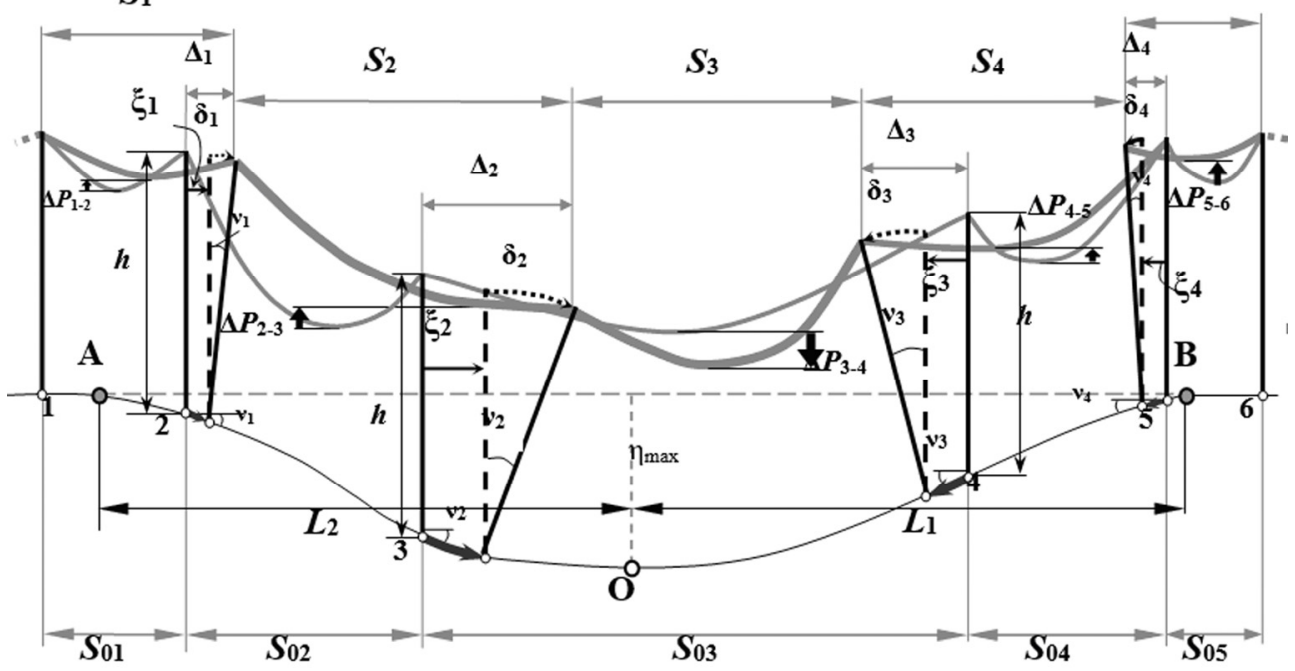

Fig. 1. Change in transmission line sag $\left(\Delta P_{2-3}, \Delta P_{3-4}, \Delta P_{4-5}, \Delta P_{5-6}\right.$, $)$ caused by horizontal displacements and slopes at tower installation sites. $\xi_{1}, \xi_{2}, \xi_{3}, \xi_{4}$ - horizontal displacement at tower installation sites. $v_{1}, v_{2}, v_{3}, v_{4}$ - tower slope angles caused by the sloping of tower installation sites. $\delta_{1}, \delta_{2}, \delta_{3}, \delta_{4}-$ tower displacement caused by slopes. $\Delta_{1}, \Delta_{2}, \Delta_{3}, \Delta_{4}$ - total tower displacement caused by slopes and horizontal displacements. $S_{01}, S_{02}, S_{03}, S_{04}, S_{05}$ - distance between wire suspension points on towers before underground mining activities. $S_{1}, S_{2}, S_{3}, S_{4}, S_{5}$ - distance between wire suspension points on towers after underground mining activities. $h$ - height of wire suspension points from the Earth's surface. $L_{1}, L_{2}-$ semi-troughs across the strike (falling and rising respectively). A, B - subsidence trough boundary. $\mathrm{O}$ - point of maximum subsidence $\left(\eta_{\max }\right)$.

The boundaries of the zone of influence of underground mining activities or underground construction - subsidence troughs - are established along the length of semi-troughs $\left(L_{1}, L_{2}, L_{3}\right.$ in Fig. 1, 2), which in turn are determined by boundary corners [1, 2]. So, boundary corners are defined according to [1] for major coal basins, and [2] for subway tunnel construction in St. Petersburg. The zone of influence of subway tunneling can be established based on modeling of geomechanical processes by finite element method (FEM). Changes in wire tension in areas between transmission towers are due to inclination and horizontal displacements of these towers, caused by slope deformations and horizontal displacements within the subsidence trough boundaries [3-13]. The combined impact of these factors determines how closer or farther away the wire suspension points have moved, and accordingly how the point with maximum wire sag in the span between towers has been lowered or lifted. Lowering of the maximum sag point entails weaker wire tension, while lifting means stronger wire tension. The mechanism for the lifting or lowering of transmission line sag, which is caused by the impact of slopes and horizontal displacements on towers, is shown schematically in Fig. 1.

A shift in the position of towers situated within subsidence trough boundaries (AOB in Fig. 1 and abcde in Fig. 2), which is caused by the cumulative effects of horizontal displacements and slopes, are defined by the following formulas:

$$
\Delta_{1}=\xi_{1}+\delta_{1}, \Delta_{2}=\xi_{2}+\delta_{2} \ldots \Delta_{j}=\xi_{j}+\delta_{j}
$$

where $\Delta_{1}, \Delta_{2}, \ldots, \Delta_{j}$ are the total displacements for towers $1,2, \ldots, j$ respectively in the zone of influence of underground mining activities (Fig. 1, 2); $\xi_{1}, \xi_{2}, \ldots, \xi_{j}$ - displacement of towers $1,2, \ldots, j$ respectively, caused by horizontal displacements; $\delta_{1}, \delta_{2}, \ldots, \delta_{j}$-displacement 
of towers $1,2, \ldots, j$ respectively, caused by slope deformations (Fig. 1).

Displacements caused by the effect of slope deformations is determined by the following formulas

$$
\delta_{1}=h \cdot i_{1}=h \cdot \operatorname{tg} v_{1}, \delta_{2}=h \cdot i_{2}=h \cdot \operatorname{tg} v_{2} \ldots \delta_{j}=h \cdot i_{j}=h \cdot \operatorname{tg} v_{j}
$$

where $h$ is the height of wire suspension point on tower from the Earth's surface; $i_{1}, i_{2}$, $\ldots, i_{j}$ are the slopes at the installation sites of towers $1,2, \ldots, j$, determined by predictive calculations and equal to the slope of the towers; $v_{1}, v_{2}, \ldots, v_{j}$-slope angles of towers 1,2 , $\ldots, j$, defined as:

$$
v_{1}=\operatorname{arctg}\left(i_{1}\right), v_{2}=\operatorname{arctg}\left(i_{2}\right) \ldots v_{j}=\operatorname{arctg}\left(i_{j}\right)
$$

The predicted values for horizontal displacements $\left(\xi_{j}\right)$ and slopes $\left(i_{j}\right)$ can be obtained either by standard curve method $[1,2]$, or by numerical modeling of deformation processes via finite element method (software package PLAXIS), or by analytical methods .

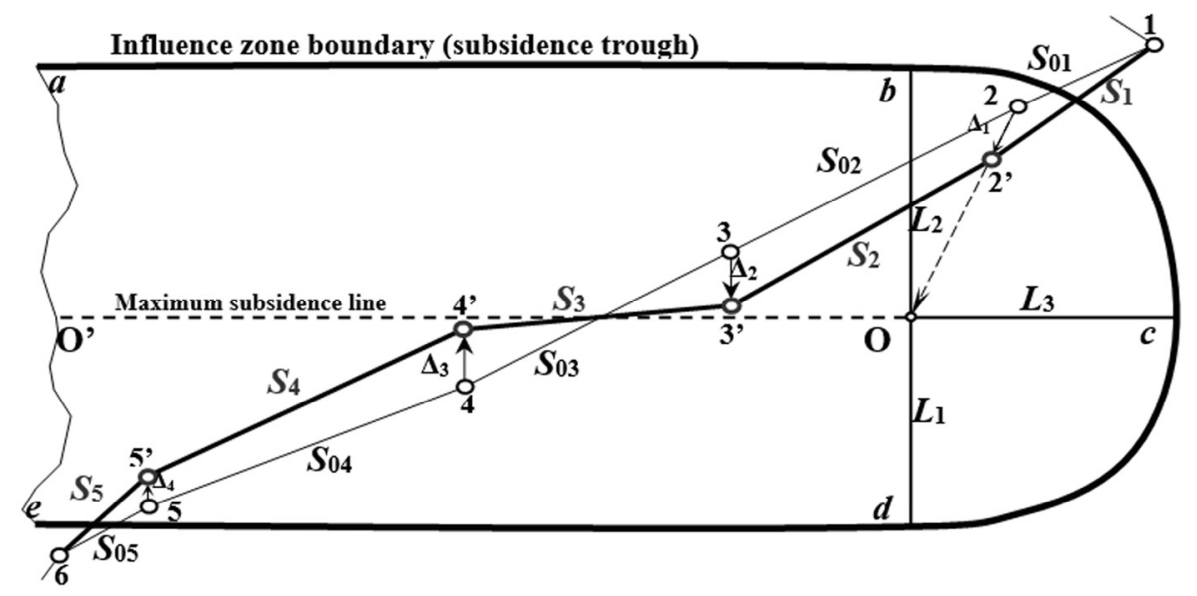

Fig. 2. Changes in distance between transmission towers located diagonally to the subsidence trough boundaries. The designations are the same as in Figure 1.

AutoCAD - a software application for computer-aided design - is used to construct the subsidence trough boundary and mark the position of power transmission towers. Next, the distances $S_{01}, S_{02}, \ldots, S_{05}$ between these transmission towers are calculated. They correspond to the distances between wire suspension points on towers $1-2,2-3,3-4,4-5,5-6$ before underground mining activities (Fig. 2). From these transmission towers located within the subsidence trough, tower displacements $\Delta_{j}$ calculated by formula (2) are laid in the direction of horizontal displacement vectors. Within subsidence trough zone abde, horizontal displacement vectors are directed towards the maximum subsidence line OO' at an angle of $90^{\circ}$ to the line. In subsidence trough zone $b c d \mathrm{O}$, these vectors are directed towards point $\mathrm{O}$ (Fig. 2). As a result, new positions for transmission towers 1', 2', 3', 4', 5' under the influence of mining activities is determined. From here, we get the distance between transmission towers $S_{1}, S_{2}, \ldots, S_{5}$, corresponding to the distance between wire suspension points on towers 1'-2', 2'-3', 3'-4', 4'-5', 5'-6' after underground mining activities (Fig. 2). Hence, the increase or decrease in the distance between wire suspension points as a result of tower displacement caused by underground mining activities is determined as follows

$$
\Delta S_{1}=S_{1}-S_{01}, \Delta S_{2}=S_{2}-S_{02} \ldots \Delta S_{j}=S_{j}-S_{0 j}
$$

If $S_{j}>S_{0 j}$, then $\Delta S_{j}>0$, that is, the sag will rise as a result of an increase in the distance between the towers. If $S_{j}<S_{0 j}$, then $\Delta S_{j}<0$, that is, the sag will fall as a result of a decrease in distance.

Next, we determine the changes in wire sag (rising and falling) using increment $\Delta S_{j}$ in the distance between wire suspension points. Fundamental calculation of the extent of change in 
wire sag is shown in Fig. 3 .

In the diagram, $S_{0}$ is the distance between towers $m$ and $n$. If this distance is reduced by $\Delta S$, the wire takes the position of $A c m$ arc with radius $r$. As a result, maximum sag $p_{m, n}$ is formed relative to $m n$ (Fig. 3), which can be defined as $p_{m, n}=O C-O D=r-r \cdot \cos \alpha=r \cdot(1-\cos \alpha)$.

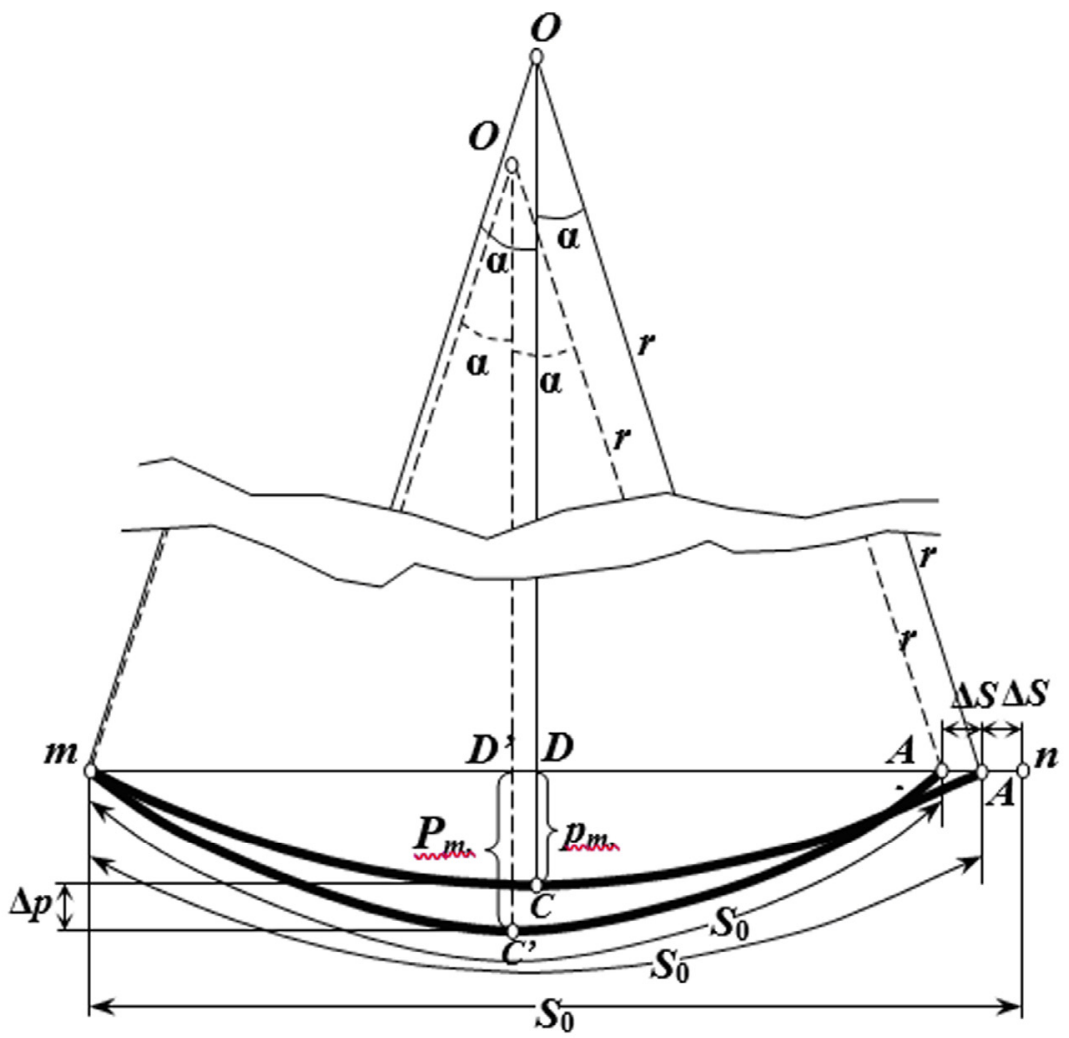

Fig. 3. Calculating the changes in wire sag between transmission towers.

$\Delta S$ is calculated as

$$
\Delta S=S_{0}-A m=2 r \cdot(\alpha-\sin \alpha)
$$

Due to the smallness of angle $\alpha$, using trigonometry formulas and expanding trigonometric functions in series, we get

$$
\Delta S=\frac{8 p_{m, n}^{2}}{3 S_{0}}
$$

From this we can determine the sag corresponding to $\Delta S$ decrease in the distance between towers $m$ and $n$ (Fig. 3):

$$
p_{m, n}=\sqrt{\frac{3 \cdot|\Delta S| \cdot S_{0}}{8}} .
$$

To predict the extent sag will increase as a result of shorter distance between towers $m$ and $n$, it is necessary to calculate the sag resulting from a $2 \Delta S$ decrease in distance between towers $m$ and $n$. The sag will be (Fig. 3):

$$
P_{m, n}=\sqrt{\frac{3 \cdot|(2 \cdot \Delta S)| \cdot S_{0}}{8}} .
$$


Then an increment or change in sag, due to shorter distance between towers $m$ and $n$ will be (Fig. 3):

$$
\Delta p=P_{m, n}-p_{m, n}
$$

So, using (6), we determine the size of wire sag between towers $1,2, \ldots, j$ (Fig. 1, 2) caused by increments $\Delta S_{1}, \Delta S_{2}, \ldots, \Delta \mathrm{S}_{j}$ (see (5)) between wire suspension points on the towers. Then, using formula (7), we determine the sag between the same transmission towers, but at double increment $\left(2 \Delta S_{1}, 2 \Delta S_{2}, \ldots, 2 \Delta S_{j}\right)$ in distances between their wire suspension points. From here, formula (8) is used to calculate the change in sag between the transmission towers $\left(\Delta p_{j}\right)$.

If for predictive calculations, we put in (6) and (7) a decrease in distance between wire suspension points (i.e., put increment $\Delta \mathrm{S}_{j}<0$ ), then the value $\Delta p_{j}$ obtained from expression (8) will represent a lowering in the wire sag between the transmission towers. If in (6) and (7), we put an increase in the distance between wire suspension points (i.e., $\Delta \mathrm{S}_{j}>0$ ), $\Delta \mathrm{p}_{\mathrm{j}}$ obtained from (8) will represent a lift in wire sag between the towers.

As a result, for transmission line sections situated in the zone of influence of underground mining activities or underground construction (tunnels, underground subway stations, reservoirs, etc..), we can highlight the greatest sag changes (lifting or lowering) in the middle of the wire span between transmission towers, which correspond to an increase or decrease in wire tension, and assess the slope angles of the towers. All these are required to ensure that tower leveling and wire re-stretching are effected quickly to prevent the cables from breaking or the middle of the wire span from lowering too much. This would enable increase the distance from the middle of the wire span to the Earth's surface to a safe minimum.

To ensure that tower leveling and wire re-stretching are carried out promptly during the influence of underground mining activities, it is necessary to carry out special monitoring surveillance over the state of transmission lines. Given the peculiarities of the object, such as its reaction to the impact of underground mining activities, the following method for monitoring the technical condition of transmission lines is proposed.

\section{Conclusions}

The above method enables one to determine the extent of wire elongation between highvoltage transmission towers without direct contact with the high-voltage wires suspended from the towers at high altitude. That is, there is no need to disconnect the power transmission line when checking the technical condition of the cables.

\section{References}

1. Rules for protection of structures and natural objects from harmful effects of underground mining activities at coal fields (VNIMI, St. Petersburg, 1998)

2. Manual for measures aimed at protecting buildings and structures from the effects of mining activities in subway construction (Stroyizdat, Leningrad, 1973)

3. Franzius J. N. PhD thesis (London, 2003)

4. French Society for Trenchless Technology. Microtunneling and Horizontal Drilling. Recommendations (FSTT, London, ISTE, 2006)

5. Ghilani, Ch. D. Adjustment Computations. Spatial Data Analysis (NJ, John Wiley \& sons, 2010)

6. Martos, F., International Strata Control Congress (Leipzig, 1958) 
7. Schmidt, B. Ph.D. Thesis (University of Illinois, Urbana, 1969)

8. V.N. Gusev, D.A. Iliukhin, A.E. Zhuravlev, Proceedings of XV International ISM Congress, 600, 2013.

9. E. Leca, Tunnelling and Underground Space Technology, 15, 119 (2006)

10. Puller, M. Deep Excavations: a practical manual (Thomas Telford, London, 2003)

11. H. Quick, J. Michael, V. Schöttner, R. Katzenbach, U. Arslan, Tunnelling and Deep Excavation in Soft Ground, 1998

12. W. J. Rankin, Engineering geology of underground movements, 20, 79 (1988)

13. B. Schmidt, Ph.D. Thesis (University of Illinois, Urbana, 1969) 